\title{
Review
}

\section{Spasticity after spinal cord injury}

\author{
MM Adams ${ }^{1}$ and AL Hicks*,1 \\ ${ }^{1}$ Department of Kinesiology, McMaster University, Hamilton, Ontario, Canada
}

\begin{abstract}
Symptoms of spasticity are often experienced by individuals with spinal cord injury (SCI) following a period of spinal shock and, in many cases, these symptoms negatively affect quality of life. Despite its prevalence, spasticity as a syndrome in the SCI population is not always managed effectively. This is likely due to the fact that the syndrome can have various presentations, each with their own specific etiology. This overview summarizes the symptoms and pathophysiology of the various presentations of spasticity in the SCI population and discusses the currently accepted management techniques. There is a need for a better understanding of the syndrome of spasticity as well as the development of a valid and reliable assessment tool.
\end{abstract}

Spinal Cord (2005) 43, 577-586. doi:10.1038/sj.sc.3101757; published online 19 April 2005

Keywords: spinal cord injury; spasticity; pathophysiology; management; definition; prevalence

\section{Introduction}

Unequivocally, 'spasticity' is understood to be among the symptoms resulting from injury to the upper motor neurons within the central nervous system (CNS) and is a common but not an inevitable sequelae of spinal cord injury (SCI). ${ }^{1-3}$ The most commonly cited definition for spasticity is that published by Lance in $19800^{4}$ 'Spasticity is a motor disorder characterized by a velocitydependent increase in tonic stretch reflexes (muscle tone) with exaggerated tendon jerks, resulting from hyperexcitability of the stretch reflex, as one component of the upper motoneuron syndrome'. There remains, however, discrepancy in the literature about the definition of spasticity; whereas some authors include symptoms such as clonus, hyperactive tendon reflexes, and spasms within the umbrella term 'spasticity', 1,5-7 others discuss these same symptoms as related to but separate from spasticity, which is defined by these authors as increased muscle tone..$^{3,8-11} \mathrm{Decq}^{2}$ recently has suggested the use of a modified definition, whereby spasticity, in general, is defined as a symptom of the upper motor neuron syndrome characterized by an exaggeration of the stretch reflex secondary to hyperexcitability of spinal reflexes. He follows by separating the various components of spasticity into sub-definitions: (1) intrinsic tonic spasticity: exaggeration of the tonic component of the stretch reflex (manifesting as increased tone), (2) intrinsic phasic spasticity: exaggeration of the phasic component of the stretch reflex

*Correspondence: AL Hicks, Department of Kinesiology, McMaster University, 1280 Main Street West, Hamilton, Ontario, Canada L8S 4K1 (manifesting as tendon hyper-reflexia and clonus), and (3) extrinsic spasticity: exaggeration of extrinsic flexion or extension spinal reflexes. Throughout the discussion to follow, the modified definition of spasticity suggested by $\mathrm{Decq}^{2}$ will be utilized in order to clearly differentiate between the various spasticity-related symptoms that are experienced by individuals with SCI.

The literature has shown that $65-78 \%$ of sample populations of individuals with chronic SCI $(\geqslant 1$ year postinjury) have symptoms of spasticity. ${ }^{6,12}$ Although unclear, it has been suggested that the American Spinal Injury Association (ASIA) classification of SCI (severity) and level of injury may predict the likelihood of developing spasticity; for example, in individuals with cervical SCI, 93\% of those diagnosed as ASIA A and $78 \%$ of those diagnosed as ASIA B-D reported having symptoms of spasticity, whereas in individuals with thoracic SCI, $72 \%$ of those diagnosed as ASIA A and $73 \%$ of those diagnosed as ASIA B-D reported symptoms of spasticity. ${ }^{6}$ The greater incidence of lower motor neuron injury associated with lower-level injuries results in a reduced tendency for spasticity development in these individuals. ${ }^{6,12}$ Whereas the resolution of spinal shock may coincide with an increase in spasticity symptoms,${ }^{12}$ there is no clear relationship between the presence of spasticity symptoms and time since injury beyond the spinal shock period. ${ }^{6}$

Spasticity has the potential to negatively influence quality of life (QOL) through restricting activities of daily living (ADL), inhibiting effective walking and selfcare, causing pain and fatigue, disturbing sleep, compromising safety, contributing to the development of 
contractures, pressure ulcers, infections, negative selfimage, complicating the role of the caretaker, and impeding rehabilitation efforts. ${ }^{3,6,7,13-17}$ Reports of problematic spasticity 1, 3, and 5 years following SCI occurred in $35, \sim 31$, and $\sim 27 \%$ of a sample of a population-based cohort of SCI survivors reported to the Colorado Spinal Cord Injury Early Notification System. ${ }^{18}$ Similarly, of those individuals reporting spasticity in the Stockholm Spinal Cord Injury Study, $40 \%$ reported their spasticity to be problematic, in that ADL were restricted and/or the spasticity caused pain. ${ }^{19}$ In a study by Sköld et $a{ }^{6},{ }^{6} 20$ and $4 \%$ of their total sample perceived their spasticity to restrict ADL and cause pain, respectively. Although Krawetz and Nance ${ }^{20}$ have identified that severity of spasticity is among the factors that can reduce the degree to which walking is effective in functional ambulators after SCI, Norman et $a l^{21}$ have emphasized that, despite the common association between spasticity and clinical signs of abnormal gait, the nature of this relationship remains unclear. Furthermore, it must be noted that, although spasticity can have a negative impact on QOL, it has been suggested that symptoms of spasticity may increase stability in sitting and standing, facilitate the performance of some ADL and transfers, increase muscle bulk and strength of spastic muscles (thereby helping prevent osteopenia), and increase venous return (possibly diminishing the incidence of deep vein thrombosis). ${ }^{10,14-16}$ This potential for a beneficial effect of spasticity on QOL has a large impact upon decisions regarding its management. 7,16

The use of varied definitions of spasticity complicate its valid and reliable assessment. ${ }^{14,22}$ While the Ashworth and modified Ashworth scales ${ }^{14,15,23}$ are commonly used to assess the severity of spasticity, there is some question about their validity in the lower limbs of persons with SCI. ${ }^{24}$ As spasticity outcomes vary between clinical patient groups and depend on a variety of factors within each individual, a battery of assessment tools is recommended, incorporating clinical, electrophysiologic, neurophysiological, ${ }^{25}$ and/or biomechanical techniques. ${ }^{14,26,27}$ It is important to note that there are generally poor correlations among clinical scales and, further, reductions in spasticity are not necessarily correlated with improvement in function. ${ }^{27-29}$ The lack of agreed-upon measures of spasticity as a whole or of the various 'components of spasticity' limits the quantification of physical status and the study of effectiveness of management strategies. ${ }^{27}$ The ideal scale should not only quantify the degree and nature of the spasticity, but patient satisfaction, global function, and technological assessment should be considered. ${ }^{29}$

\section{Pathophysiology of spasticity in SCI}

In general, spasticity is classified as a symptom of the upper motor neuron syndrome, characterized by an exaggeration of the stretch reflex secondary to hyperexcitability of spinal reflexes. ${ }^{2}$ Upper motor neurons originate in the brain and brain stem and project to lower motor neurons within the brain stem and spinal cord. ${ }^{11}$ The lower motor neurons are of two types, both of which originate in the ventral horn of the spinal cord: (1) alpha motor neurons project to extrafusal skeletal fibers and (2) gamma motor neurons project to intrafusal muscle fibers within the muscle spindle. ${ }^{11}$ With a lesion of the CNS comes interruption of the signals sent via the upper motor neurons to the lower motor neurons or related interneurons. Immediately following SCI, a period exists whereby the individual presents with flaccid muscle paralysis and loss of tendon reflexes below the level of the lesion. ${ }^{5}$ This period was first described in 1750, with the term 'spinal shock' introduced by Marshall Hall in $1850 .{ }^{30}$ Spinal shock has been reported to end from 1 to 3 days $^{31}$ to a few weeks postinjury, with the gradual development of exaggerated tendon reflexes, increased muscle tone, and involuntary muscle spasms: ${ }^{5}$ the symptoms of spasticity. Recent animal research suggested that a recovery of relatively normal motor neuron excitability and plateau potential behavior (sustained depolarizations), in the absence of normal inhibitory control to turn off plateaus and associated sustained firing, may be implicated in the recovery of spinal shock following $\mathrm{SCI}{ }^{32}$

\section{Intrinsic tonic spasticity}

Decq $^{2}$ has differentiated intrinsic tonic spasticity (increased muscle tone) as that component of spasticity resulting from an exaggeration of the tonic component of the stretch reflex. Briefly, the stretch reflex is a monosynaptic reflex pathway that originates in the muscle spindles embedded parallel to the muscle fibers and travels via a Ia afferent to the spinal cord, where it synapses either first with interneurons or directly with an alpha motor neuron innervating the muscle from which the stimulus originated. ${ }^{11}$ The tonic component of the stretch reflex associated with increased muscle tone results from a maintained stretch of the central region of the muscle fibers and the reflex is polysynaptic. ${ }^{11}$ Upon a sustained stretch, both type Ia and type II afferents (from secondary spindle endings) synapse with interneurons within the ventral horn of the spinal cord. Synapses of the interneurons with alpha motor neurons facilitate contraction in the muscle being stretched. ${ }^{11}$

It is the hyperexcitability of this tonic stretch reflex that is commonly thought to result in increased muscle tone in response to passive stretch following SCI. ${ }^{3}$ This hypertonia is velocity-dependent, with faster stretching velocities being associated with greater amounts of reflex activity. $^{3}$ The development of tonic stretch reflex hyperexcitability could be due to a lower threshold, an increased gain of the stretch reflex, or a combination of the two. ${ }^{22}$ The resultant increase in muscle tone is thought to be due to a combination of increased denervation hypersensitivity $2,3,5,9,33$ and changed muscle properties. $^{11,13,22,34,35}$ Denervation leads to an initial downregulation of neuronal membrane receptors, followed by an upregulation, with enhanced sensitivity to neurotransmitters. ${ }^{2}$ Gradual changes in muscle proper- 
ties also occur following SCI, such as fibrosis, atrophy of muscle fibers, decrease in the elastic properties, decrease in the number of sarcomeres, accumulation of connective tissue, and alteration of contractile properties toward tonic muscle characteristics, which likely contribute to the increased passive tension. ${ }^{11,13,22,34-36}$

\section{Intrinsic phasic spasticity}

Intrinsic phasic spasticity encapsulates symptoms such as tendon hyper-reflexia and clonus, and is due to exaggeration of the phasic component of the stretch reflex. ${ }^{2}$ Tendon hyper-reflexia is identified as an exaggerated muscle response to an externally applied tap of deep tendons. ${ }^{7}$ Reduced presynaptic Ia inhibition is thought to play an important role in this hyperreflexia, as the occurrence of reduced presynaptic inhibition of group Ia fibers appears to correlate with the excitability of tendon reflexes. ${ }^{36}$

Clonus has been defined as involuntary rhythmic muscle contraction that can result in distal joint oscillation, $^{37}$ and most often occurs at the ankle. ${ }^{2,7,9}$ Clonus is elicited by a sudden rapid stretch of a muscle. ${ }^{38}$ The prevailing theory explaining the underlying mechanism responsible for clonus is that of recurrent activation of stretch reflexes. ${ }^{11,37,38}$ According to this theory, dorsiflexing the ankle causes activation of the Ia muscle spindle afferents and induces a reflex of the triceps surae, resulting in plantar flexion of the ankle. ${ }^{11,37,38}$ This reflex contraction is brief, essentially phasic, and ceases rapidly. ${ }^{2}$ The muscle then relaxes, causing the ankle to be dorsiflexed once again, due either gravity or the stretch being sustained by an examiner. $^{2}$ The result is a new stretch reflex, etc. ${ }^{2,37}$ Ultimately, it is the disinhibition of the stretch reflex due to interruption of descending influences with SCI, that is thought to cause exaggeration of the phasic stretch reflex pathway and, hence, clonus. ${ }^{3}$

The second theory is that clonus is the result of activity of a central oscillator or generator within the spinal cord, which rhythmically activates alpha motor neurons in response to peripheral events. ${ }^{37,38}$ Beres-Jones et $a l^{37}$ outline observations that they feel support such a hypothesis: (1) reports of similar frequencies of clonus among ankle, knee, and wrist muscles, (2) observations that the clonus frequency is not entrained by the input frequency, suggesting that clonus cannot be solely stretchmediated, (3) the finding that stimuli other than stretch evoke clonus, and (4) the observation of a refractory period following the clonic EMG burst where tendon tap, H-reflex stimulation, and vibration fail to elicit an efferent response. Therefore, whereas reduced presynaptic inhibition of group Ia fibers appears to be among the contributing factors to tendon hyper-reflexia, the underlying mechanism of clonus has not been clearly elucidated.

\section{Extrinsic spasticity}

In addition to the various intrinsic factors that contribute to symptoms of spasticity, involuntary muscle spasms can also occur in response to a perceived noxious stimulus originating extrinsic to the muscle: extrinsic spasticity. ${ }^{2,3,7}$ Flexion spasms are the most common form of extrinsic spasticity, triggered by afferent input from skin, muscle, subcutaneous tissues, and joints (collectively referred to as 'flexor reflex afferents'). These flexor reflex afferents mediate the polysynaptic reflexes involved in the flexion withdrawal reflex. 3,35,39 SCI can interrupt the inhibition of these reflexes by supraspinal pathways, making them hyperexcitable. ${ }^{2,3,40}$ In other words, whereas flexor withdrawal reflexes occur normally in individuals without SCI, upon disruption of normal descending influences, the threshold for the flexor withdrawal reflex may become lowered, the gain of the system may become raised, or both may occur together. ${ }^{3}$ A recent study has provided evidence to implicate plateau potentials in the spinal interneuronal and motoneuronal circuitry in the hyperexcitability of flexion withdrawal reflexes in individuals with chronic SCI. ${ }^{41}$ Intrasegmental polysynaptic connections cause the flexor reflex initiated by a localized stimulus to generate a widespread flexor spasm, which can appear as a coordinated flexion of all joints of the leg. ${ }^{35,39}$

\section{Management of spasticity following SCI}

In contrast to the general lack of agreement within the literature about the definition and evaluation of spasticity, there appears to be widespread agreement that decisions regarding the management of spasticity must be based on the goal of achieving balance between the useful and detrimental effects of spasticity on an individual's QOL. ${ }^{2,10,16,34}$ The management of spasticity may be desired for the reduction of 'passive problems', such as preventing contracture, reducing pain, facilitating splint wearing, easing positioning and hygiene, and preventing contractures, or of 'functional problems', including the individual's reduced ability to perform useful work with the motor system. ${ }^{9}$

In general, no one treatment option will successfully manage spasticity in all individuals; the most conservative tactics are utilized first, with a progression from physical rehabilitation modalities, pharmacologic interventions, injection techniques, intrathecal baclofen, and lastly, surgery. ${ }^{15}$ In general, local treatments are used primarily by individuals with spasticity predominating in only certain muscle groups, such as which occurs mainly in individuals with stroke or traumatic brain injury. ${ }^{42}$ In the case of SCI, the distribution of spasticity tends to be more diffuse, making regional or systemic treatment preferable. $^{34}$ The decision whether or not to treat spasticity and, if so, in what manner, is summarized nicely in a flow chart by Parziale et al (Figure $1^{16}$ ).

\section{Conservative/physical rehabilitation management}

It is generally agreed that physical therapy/rehabilitation is an essential component in the management 


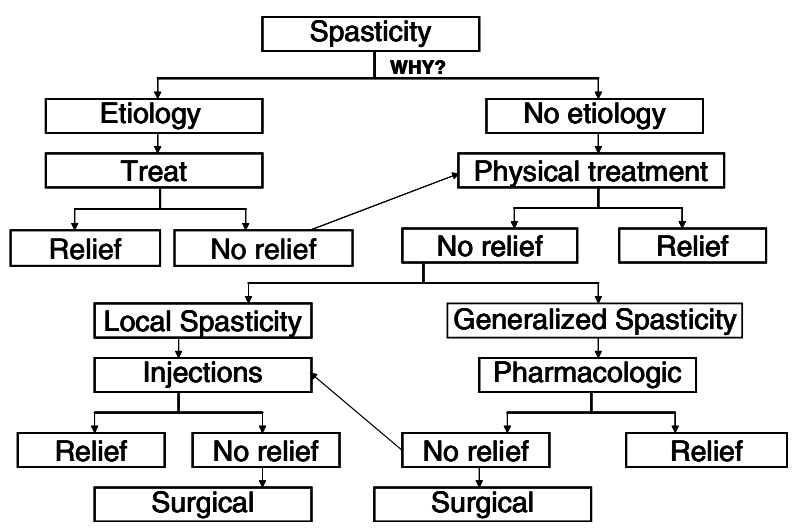

Figure 1 Spasticity management (adapted from Parziale et $a l^{16}$ )

of spasticity as a first line of defence, as well as in a long-term regimen during and after the implementation of pharmacological or surgical strategies. ${ }^{10,17,36}$ The goal of physical therapy is to diminish spasticity in order to allow expression of voluntary mobility and movements and/or to improve the comfort and independence in tasks related to QOL, such as transfers, dressing, and using the washroom. ${ }^{43}$ The literature on the conservative/physical treatment of spasticity is sparse, and some have questioned the effectiveness of these management strategies. ${ }^{36}$ Table 1 summarizes the most common physical therapy approaches to spasticity management.

\section{Pharmacologic management}

Systemic pharmacological treatments of spasticity symptoms are often prescribed in the SCI population. ${ }^{34}$ Most antispasticity medications can be grouped roughly into three functional categories: (1) 'GABAergic' drugs that act at interneurons that use the neurotransmitter gamma-aminobutyric acid (GABA) in the CNS (eg, baclofen and diazepam), (2) alpha-2-adrenergic those that act at alpha-2 receptors in the CNS (eg, tizanidine and clonidine), and (3) peripheral acting those that act at the neuromuscular level (eg, dantrolene).$^{48}$ Numerous factors are taken into consideration when the physician and patient are deciding upon the role of pharmacologic management, including time since injury, onset of spasticity, severity, prognosis, available support system, cognitive status, concurrent medical problems, geographic location, symptom location, and financial issues. ${ }^{9}$ Each type of medication has potentially serious side effects and no single medication has a beneficial effect in all individuals. ${ }^{15,34}$

\section{Diazepam}

GABA is an inhibitory neurotransmitter typically found in short interneurons. ${ }^{49}$ There are two types of GABA receptors, with diazepam acting at the $\mathrm{GABA}_{\mathrm{A}}$ receptor, the more prominent of the two. ${ }^{34}$ When GABA is released from a presynaptic membrane and binds to $\mathrm{GABA}_{\mathrm{A}}$ receptors on the postsynaptic membrane, chloride channels are opened, allowing chloride ions to flow into the postsynaptic membrane, hyperpolarizing the membrane. ${ }^{34}$ As a result, GABA inhibits action potential transmission. Presynaptic inhibition occurs when a GABAergic interneuron connects with the terminal of a Ia afferent and, therefore, decreases the excitability of the Ia afferent terminal and causes a decrease in the transmitter released from the Ia afferent to, ultimately, the motor neuron. ${ }^{34}$

Diazepam is the most commonly used agent among the benzodiazepines to treat spasticity, which, as a group, acts by enhancing the efficiency of GABAergic transmission. ${ }^{9,10,13,48}$ Diazepam does not directly mimic GABA. $^{15,34}$ Rather, it binds postsynaptically near $\mathrm{GABA}_{\mathrm{A}}$ receptors, facilitates GABA-mediated chloride conductance and, therefore, hyperpolarizes the membrane. ${ }^{9,10,15,48}$ The result of diazepam administration is an increase in presynaptic inhibition of afferent neuronal terminals and, thus, a reduction of monosynaptic and polysynaptic reflexes. ${ }^{9,13,34}$ Diazepam is often mentioned as being most effective in the treatment of hyperactive reflexes and painful spasms in individuals with SCI (compared to individuals with stroke or multiple sclerosis). ${ }^{9,10,13,15}$ Functional measures, however, have been shown not to improve with diazepam treatment. ${ }^{9}$ Clonazepam, another benzodiazepine, causes less sedation than diazepam and has slightly lower risk for dependence; it is typically used for the reduction of night-time spasms. ${ }^{15,34}$

\section{Baclofen}

Unlike diazepam, baclofen is a structural anologue of GABA and an agonist of GABA $_{B}$ receptors. ${ }^{9,10,13,15,48}$ When baclofen binds to $\mathrm{GABA}_{\mathrm{B}}$ receptors both presynaptically and postsynaptically, monosynaptic and polysynaptic spinal reflexes are inhibited. ${ }^{9,10,13,15,44}$ Upon binding of baclofen presynaptically, influx of calcium into the presynaptic terminal is restricted and neurotransmitter release in excitatory spinal pathways is decreased, leading to a decrease in alpha motor neuron activity. $9,10,15,34,49$ When baclofen binds to $\mathrm{GABA}_{\mathrm{B}}$ receptors on the postsynaptic membrane of a Ia afferent, potassium channels allow the flow of potassium out of the Ia afferent terminal, resulting in membrane hyperpolarization and, hence, interruption of action potential transmission. ${ }^{9,10,34}$

Baclofen is a commonly used drug for spasticity in the SCI population. ${ }^{21}$ It has been reported to be particularly effective for reducing flexor spasms. ${ }^{9,10,34}$ However, the literature also suggests that baclofen may have no positive effect on walking ability or the performance of ADL. ${ }^{9,21,34,48}$ Similar to other muscle relaxants, baclofen may impair the ability of the patient to walk or stand. ${ }^{13,15}$ Baclofen has been shown to be safe and effective for long-term use, with no evidence for tolerance. $^{15}$ 
Table 1 Physical techniques in the management of spasticity

Physical therapy technique description and comment on effectiveness

\section{Positioning ${ }^{15,43}$}

- In bed and during sitting

- Reports of clinical effectiveness; impact remains to be proven scientifically ${ }^{14}$

\section{Range of motion/stretching}

- Includes passive stretch and passive lengthening ${ }^{14,43}$

- Benefits may carry over for several hours ${ }^{15,16}$

- Effects remain to be quantified and the efficacy remains to be determined despite the clinical evidence for the benefits $^{14}$

\section{Weight-bearing}

- Using a tilt table or standing frame

- Benefits are greater than stretching alone and may persist into next day ${ }^{8}$

- Effectiveness has been questioned ${ }^{14}$

\section{Muscle strengthening}

- Progressive addition of resistance to muscles with voluntary control ${ }^{16,43}$

\section{Electrical stimulation}

- Various methods: stimulation to the antagonist muscle, application of tetanic contraction to the spastic muscle, functional electrical stimulation (FES), and transcutaneous electrical nerve stimulation (TENS) $^{14-16,43}$

- Reports of beneficial effects between only $10 \mathrm{~min}$ and $3 h^{14,15,43}$
Purpose/suggested mechanisms

- Important to the maintenance of muscle length ${ }^{15,43}$

- Prevents contractures ${ }^{42,43}$

- Causes temporary reduction in intensity of muscle contraction in reaction to muscle stretch ${ }^{43}$

- May cause plastic changes within the central nervous system and/or mechanical changes at the muscle, tendon, and soft-tissue level ${ }^{14}$

- Prolonged stretch of ankle plantar flexor muscles $8,14,15$

- Mechanism remains uncertain; suggested to include a modulating influence from cutaneous and joint receptor input to the spinal motor neurons, resulting in decreased excitability $^{8}$

- Emphasis of balance of agonist and antagonist groups of muscles with voluntary control ${ }^{16,43}$

- Stimulation of the antagonist muscle: augmentation of reciprocal inhibition of the spastic muscle ${ }^{14}$

- Repetitive tetanic stimulation of spastic muscle: fatigue of the muscle due to repetitive tetanic stimulation ${ }^{14}$

- FES: change the mechanical properties of a spastic joint by strengthening the antagonists of the spastic muscle or might decrease the hyperactivity of spastic muscles through reciprocal inhibition ${ }^{54}$

- TENS: may involve the stimulation of large diameter afferent fibers that travel from mechanoreceptors to the spinal cord ${ }^{14}$

- May involve the activation of inhibitory networks within the spinal cord $^{56}$

- More strongly affected patients require stronger stimuli and/or higher frequencies ${ }^{56}$

- Cold: may cause slowing of nerve conduction, decrease in sensitivity of cutaneous receptors, and alteration of CNS excitability ${ }^{14-16}$

- Heat: facilitation of uptake of released neurotransmitters and return of calcium to the sarcoplasmic reticulum ${ }^{16}$

- Enables long-term stretch $^{42,43}$

- Joint can be maintained in a position that does not elicit ${\text { a } \text { spasm }^{15}}^{15}$ 


\section{Clonidine}

Clonidine is a centrally acting alpha-2 adrenergic agonist commonly used to treat hypertension.9,34 Alpha-2 receptors are located, among other regions, on presynaptic nerve terminals in the CNS and are termed adrenergic because they are involved in release of norepinephrine and acetylcholine. ${ }^{49}$ Generally speaking, an alpha-2-adrenergic agonist can bind to alpha-2 receptors, thereby preventing the normal action of norepinephrine to act as a neurotransmitter. ${ }^{49}$ Therefore, clonidine can act spinally to reduce spasticity by enhancing alpha-2-mediated presynaptic inhibition of sensory afferents, thereby suppressing spinal polysynaptic reflexes. 9,15 Clonidine has been found to be associated with improved walking ability in individuals with incomplete SCI (eg, longer cycles, increased treadmill speed, and more upright posture). ${ }^{21,50}$

\section{Tizanidine}

Tizanidine is an imidazole derivative and, like clonidine, is a centrally acting (spinally and supraspinally) alpha-2adrenergic agonist. $9,10,13,34,48$ Therefore, it acts by inhibiting the release of excitatory amino acids from the presynaptic terminals of excitatory spinal neurons. 9,10 It may also facilitate the inhibitory neurotransmitter glycine. ${ }^{9,10,13}$ Tizanidine has been shown to reduce muscle tone and frequency of muscle spasms in individuals with SCI, but no increase in functional measures has been noted. ${ }^{9,10,13,15,34}$ Manual muscle testing while being treated with tizanidine has indicated that strength is not decreased, ${ }^{10,15}$ although weakness has been reported. ${ }^{34}$

\section{Dantrolene sodium}

Dantrolene sodium is the only oral medication that acts peripherally at the muscle tissue, rather than at the spinal cord level, to weaken muscles that are overexcited. $^{9,15,34}$ It is a hydantoin derivative that inhibits muscle action potential-induced release of calcium from the sarcoplasmic reticulum to the active myosin fibers during muscle contraction by increasing the binding of calcium to the sarcoplasmic reticulum. ${ }^{9,10,13,15,34,48}$ The result is interference with excitation-contraction coupling that is necessary to produce muscle contraction. ${ }^{10,44}$ It has also been suggested that dantrolene may alter muscle spindle sensitivity by acting on the gamma motor neurons. ${ }^{9,34}$ Dantrolene appears to have a greater effect on phasic than on tonic stretch reflexes and on fast twitch rather than slow twitch muscle fibers, with the clinical significance of these discrepancies remaining unclear. ${ }^{9,10,15,34}$ There is evidence that individuals with SCI respond well to dantrolene sodium, with possible reductions in muscle tone, tendon reflexes, and clonus, and increases in range of motion. 9,34 Improvement in performance of ADL is less evident. ${ }^{34}$ Individuals with SCI are rarely treated with dantrolene, likely because its peripheral site of action results in its most common adverse effect: muscle weakness. ${ }^{9,10,13}$

\section{Cyproheptadine}

Although less commonly reported among the drugs used to treat spasticity, cyproheptadine has been associated with an improvement in the walking pattern of individuals with SCI (eg, reduced need for manual assistance, increased treadmill speed, and reduced ankle clonus). ${ }^{21,51,52}$ Cyproheptadine is a histamine and a serotonin antagonist, which is proposed to reduce spasticity via inhibition of motor neurons by 'neutralizing the spinal and supraspinal seratoninergic excitatory inputs. 34

\section{Cannabis}

Tetrahydrocannabinol (THC), available in the drug dronabinol, is the main active ingredient in cannabis. ${ }^{15}$ Cannabinoids have been shown to have efficacy in treating spasticity and are currently being studied. ${ }^{9}$ Anecdotal reports by individuals with SCI have also revealed a beneficial effect of marijuana on the management of spasticity. ${ }^{34}$ Some literature supports the hypothesis that the relaxing effect of marijuana on muscles in patients with SCI-related spasticity is due to an antispastic effect, perhaps inhibition of polysynaptic reflexes, rather than simply due to a general relaxation response. $^{34,48}$

\section{Pharmacologic-induced negative side effects}

Although pharmacologic agents have been shown to be effective in the reduction of spasticity symptoms, the overall QOL of the individual may become hampered by unwanted pharmacologic-induced side effects. Briefly, these side effects vary between pharmacologic agents and may include sedation, drowsiness, insomnia, fatigue, nausea, diarrhea, dry mouth, muscle weakness, ataxia, dizziness, hypotension, depression, impaired memory and attention, hallucinations, liver toxicity, and possible addiction. $9,10,13,15,34$ Furthermore, abrupt cessation of certain agents, particularly diazepam and baclofen, may lead to anxiety, agitation, restlessness, irritability, tremor, muscle fasciculation and twitching, nausea, hypersensitivity to touch, taste, smell, light, and sound, hallucinations, insomnia, nightmares, and seizures. $^{10,13,16,34}$

\section{Intrathecal administration of baclofen}

Intrathecal administration of baclofen combines the pharmacologic administration of baclofen with a surgical technique. ${ }^{15}$ In individuals who do not respond to oral administration of medications or to other techniques or who have had intolerable side effects from medications, intrathecal baclofen may be indicated and should be considered prior to surgical intervention. ${ }^{10,15,34}$ Briefly, a pump with reservoir $(\sim 4$ in in diameter) is surgically implanted in the subcutaneous tissue of the abdominal wall, allowing the direct delivery of the drug to the cerebrospinal fluid. ${ }^{10,15,34} \mathrm{~A}$ percutaneous puncture into the access port allows access to the 
pump reservoir. ${ }^{15}$ The dose and flow rate of baclofen are individualized through external computer communication with a computer chip within the pump. ${ }^{15}$

Bypassing the blood-brain barrier allows as much as four times the concentration of baclofen to be delivered to the spinal cord with only $1 \%$ of the oral dose. ${ }^{10,15}$ One of the main advantages is the reduction in negative systemic side effects as compared to oral administration. $^{15}$ The effectiveness of intrathecal baclofen as an anti-spasticity management therapy has been shown in individuals with SCI, with little data on functional improvements or QOL being reported. ${ }^{17,34,44,53}$ Recent reviews have described the effects of intrathecal baclofen on reducing hypertonus, spasm frequency, reflex intensity and/or spasticity-related discomfort, as well as improving QOL by allowing the individual to eat, feel, and look better and by facilitating transfers, nursing care, sleep, and, in some, walking ability. ${ }^{17,54}$

The long-term effects of treatment with intrathecal baclofen are not yet known. ${ }^{17}$ Possible complications as a result of the surgical implantation of the pump include dislodgement, disconnection, migration, catheter kinking, blockage, pump failure, battery depletion, infection, and accidental overdose. ${ }^{10,15}$ As with oral baclofen, drowsiness, dizziness, nausea, hypotension, headache, weakness, and withdrawal syndrome are possible side effects. $^{15}$

\section{Injection techniques - chemodenervation agents}

Injection for the purpose of local chemodenervation is one of the four possible routes of administration of a pharmacologic agent (enteral, transdermal, and intrathecal administration being the other three). ${ }^{9}$ The technique actually treats the upper motor neuron syndrome by simulating a lower motor neuron lesion. ${ }^{9}$ Injection techniques are preferred for treatment of focal spasticity and when agonist muscles have the functional strength once freed from antagonist spasticity. ${ }^{9,15}$ One of the benefits of injection is the minimization of systemic side effects. ${ }^{9,15}$ The injections can be applied as nerve blocks or motor point blocks, which are temporary, or as chemical neurolysis, which permanently destroys a portion of the nerve; whether an injection results in temporary or permanent chemodenervation depends on the concentration of agent administered. $^{42}$ The chemodenervation agents used include phenol, ethanol, and, more recently, botulinum toxin. 9

\section{Phenol and ethanol injections}

Local injections of phenol or ethanol are utilized less commonly in individuals with SCI; for a detailed review, see Gracies et al. ${ }^{42}$ Briefly, administration of phenol or ethanol to the nerve trunk causes short-term effects similar to a local anesthetic: blocking of sodium channels reduces nerve depolarization. ${ }^{16}$ The mechanism of the longer-term nerve block involves denaturing of protein and fibrosis of neural tissue, causing disruption of nerve conduction and interruption of the reflex arc and, hence, muscle relaxation. ${ }^{14-16,42}$ Recovery is variable between individuals, from a few days to months, as axons regenerate and reach motor end plates (Wallerian degeneration and regeneration). ${ }^{14,16,42} \mathrm{~A}$ number of factors may influence the duration of the effects, including the concentration and volume used for injection, the site of the block, vascular complications, cutaneous side effects, excessive motor weakness, sensory loss, wound infection, treatment variables after the block, and systemic side effects. ${ }^{42}$ A progressive reduction in motor unit activity tends to occur with repeated injections, however, as there is some permanent denervation with every injection. ${ }^{17}$

There have been fewer reports of adverse effects of ethanol injection in comparison to injection of phenol. ${ }^{42}$ Among the complications of these are injection site pain (particularly when intramuscular), vascular complications (phlebitis), permanent nerve damage, skin irritation, acute systemic effects (tremor, convulsions, CNS depression, and cardiovascular collapse), chronic dysesthesia, tissue necrosis, sensory dysesthesia, postblock pain due to an incomplete block, and muscular weakness. $^{14-17,42}$ Most individuals who undergo a phenol or an ethanol injection have preservation of motor strength. 42

\section{Botulinum toxin}

Botulinum toxin is the most potent neurotoxin known to man and product of the anaerobic bacteria Clostridium botulinum. ${ }^{10,15,55}$ It was initially developed for clinical use in 1980 by an opthalmologist to treat involuntary contractions and spasms of the eyelid muscles and 'crossed-eyes', and was first examined formally for the treatment of spasticity in $1989 . .^{15,55}$ Seven immunologically distinct toxins have been identified (types $A-G),{ }^{10,15}$ with types A and B being currently available for treatment. ${ }^{9}$ Compared with ethanol and phenol, whose actions are mediated by their ability to denature protein at the nerve, the botulinum toxins manifest their effects at the neuromuscular junction where they inhibit the release of acetylcholine from presynaptic motor axons. $9,10,14,15$

More specifically, botulinum toxin is injected into a muscle at its end plate region and spreads throughout the muscle and fascia approximately $30 \mathrm{~mm} \cdot{ }^{10,13}$ The mechanism of action of botulinum toxin then occurs in three stages, through coordinated action of the heavy and light chain components of the toxin: (1) binding: botulinum toxin binds to the presynpatic neuron at the neuromuscular junction via the heavy chain, ${ }^{10,13,14}$ (2) internalization: the toxin is internalized into the cell by endocytosis, where the heavy chain forms a channel to allow the light chain to enter the cytosol, and (3) inhibition of acetylcholine release: the release of acetylcholine from presynaptic vesicles is inhibited. ${ }^{15}$ In step 3 , the system involved in acetylcholine exocytosis (the soluble $N$-ethlylmaleimide-sensitive fusion protein attachment protein receptor (SNARE) complex) is 
proteolytically cleaved in different critical sections by the different toxin types. ${ }^{9,15,55}$ Type A has been described as activating zinc-dependent proteolysis of SNAP-25 (a synaptosome-associated protein), whereas toxin $\mathrm{B}$ is active on synaptobrevin-2, a protein attached to the acetylcholine vesicle (involved in docking and fusion of the synaptic vesicle to the presynaptic membrane). ${ }^{9,14,15,55}$ With a disrupted SNARE complex, acetylcholine cannot be released from the presynaptic terminal, and muscle contraction is inhibited. Therefore, without affecting the synthesis of acetylcholine, botulinum toxin causes reversible chemical denervation atrophy, thereby weakening muscle. ${ }^{10,13,15}$

As a result of the complex mechanism of action, chemical denervation subject to botulinum toxin injection develops slowly over the course of 24-72 h, peaking at 2-6 weeks. ${ }^{10,13,15}$ Collateral sprouting and slow reinnervation of chemically denervated nerve terminals allows for a gradual reversal of the clinical response. ${ }^{14,15,55}$ The duration of the boltulinum toxin response can depend on a number of factors, including muscle size, the dose of the toxin administered, activity of the muscle, and perhaps factors including physiotherapy and bracing. ${ }^{14}$ In general, the duration has been reported to be between 2 and 6 months, with $\sim 3$ months being common. ${ }^{10,13-15,51}$

Botulinum toxin has recently been touted 'the pharmacological treatment of first choice for focal spasticity ${ }^{17}$ because of the evidence for its effectiveness in reducing pain and tone, and improving range of motion, function, brace tolerance, and walking ability. ${ }^{14,17}$ Although botulinum toxin is not as commonly used in individuals with generalized spasticity (such as in SCI), improvements in pain, nursing care, hygiene, comfort, and functional activities can be induced by botulinum toxin injections into isolated muscle groups. ${ }^{15}$ Botulinum toxin therapy can also be combined with other treatments to enhance rehabilitation and function. ${ }^{15}$ In general, the literature indicates that botulinum toxin injections potentially can be useful in the treatment of spasticity secondary to SCI. ${ }^{15,55,56}$

There have been very few reports of severe adverse reactions due to the injection of botulinum toxin. ${ }^{15}$ Any possible complications of botulinum toxin are often related to the blocking of acetylcholine release from parts of the autonomic nervous system by the toxin (dry mouth, reduced sweating), or to the spreading of the toxin beyond the desired area, resulting in excessive weakness (which is ultimately reversible). ${ }^{10,13-15,55}$

\section{Surgical management}

As most surgeries performed on patients with spasticity take place at the muscle or the tendon, they are useful for treatment of focal spasticity with a purpose of improving function, correcting a deformity, or for cosmetic reasons. ${ }^{14,57}$ Although there are and have been several possible surgical techniques to treat spasticity (for a review, see Chambers ${ }^{53}$ ), only those currently relevant to individuals with SCI will be discussed here.
For example, selective rhizotomy (cutting of posterior roots to interrupt the peripheral reflex arc), although shown to be encouraging for children with cerebral palsy, is not frequently used in individuals with SCI. ${ }^{13,15,53}$ Intrathecal baclofen administration, often discussed under the topic of surgical management of spasticity, ${ }^{13,15}$ has been discussed above; currently, it is considered to be the most commonly used and successful of the surgical treatments for spasticity in individuals with SCI. ${ }^{13}$

Orthopedic surgical techniques (as opposed to neurosurgical) are reserved for only selected cases. ${ }^{14} \mathrm{~A}$ tenotomy, the release of a tendon from a severely spastic muscle, might be performed in individuals with severe spasticity and without voluntary movement. ${ }^{14}$ Tendon lengthening serves to reduce the pull on spastic muscles, thereby positioning the joints at a more natural and useful angle. ${ }^{14} \mathrm{~A}$ tendon transfer, moving the tendon attachment to the bone closer to the muscle, is performed in muscles that have at least partial voluntary function, with the goal of allowing these muscles to produce useful movements. ${ }^{14}$ The mechanism of action of tendon lengthening and tendon transfers in terms of spasticity reduction is via alteration of the tension in the intrafusal muscle spindle, resulting in a decreased stimulus for further contraction and, hence, in theory, reduced spasticity. ${ }^{53}$ It has been reported, however, that the effect of tendon lengthening and tendon transfer on spasticity is variable and unpredictable. ${ }^{53}$

\section{Conclusion}

Symptoms of spasticity are experienced by the majority of individuals with SCI and are a possible contributor to reduced QOL. ${ }^{3,6,7,13-17}$ The emerging understanding of the different pathophysiologies of the various presentations of spasticity symptoms has led to a recent suggestion that distinct terminology be used for these symptoms. ${ }^{2}$ By considering intrinsic tonic spasticity, intrinsic phasic spasticity, and extrinsic spasticity as having distinct etiologies, the identification and classification of troubling symptoms becomes more specific, thereby allowing for more effective application of management strategies. Our growing understanding of spasticity in the SCI population is serving to further enhance the QOL of those who find their spasticity symptoms to be problematic; the development of valid and reliable assessment tools is now an important consideration for continued improvement in spasticity classification and management decisions.

\section{References}

1 NINDS Spinal Cord Injury Information Page 2001 Retrieved February 5, 2004 from http://www.ninds.nih.gov/ health_and_medical/disorders/sci.htm.

2 Decq P. Pathophysiology of spasticity. Neurochirurgie 2003; 49: 163-184.

3 Sheean G. The pathophysiology of spasticity. Eur J Neurol 2002; 9(Suppl 1): 3-9. 
4 Lance JW. The control of muscle tone, reflexes, and movement: Robert Wartenberg Lecture. Neurology 1980; 30: $1303-1313$

5 Dietz V. Spastic movement disorder. Spinal Cord 2000; 38: 389-393.

6 Sköld C, Levi R, Seiger A. Spasticity after traumatic spinal cord injury: nature, severity, and location. Arch Phys Med Rehabil 1999; 80: 1548-1557.

7 St George CL. Spasticity. Mechanisms and nursing care. Nurs Clin North Am 1993; 28: 819-827.

8 Bohannon RW. Tilt table standing for reducing spasticity after spinal cord injury. Arch Phys Med Rehabil 1993; 74: $1121-1122$.

9 Elovic E. Principles of pharmaceutical management of spastic hypertonia. Phys Med Rehabil Clin N Am 2001; 12: 793-816 vii.

10 Kita M, Goodkin DE. Drugs used to treat spasticity. Drugs 2000; 59: 487-495.

11 Lundy-Ekman L. Neuroscience Fundamentals for Rehabilitation. W.B. Saunders Company: Toronto 2002.

12 Maynard FM, Karunas RS, Waring. Epidemiology of spasticity following traumatic spinal cord injury. Arch Phys Med Rehabil 1990; 71: 566-569.

13 Burchiel KJ, Hsu FP. Pain and spasticity after spinal cord injury: mechanisms and treatment. Spine 2001; 26: S146-S160.

14 Jozefczyk PB. The management of focal spasticity. Clin Neuropharmacol 2002; 25: 158-173.

15 Kirshblum S. Treatment alternatives for spinal cord injury related spasticity. J Spinal Cord Med 1999; 22: 199-217.

16 Parziale JR, Akelman E, Herz DA. Spasticity: pathophysiology and management. Orthopedics 1993; 16: 801-811.

17 Ward AB. Long-term modification of spasticity. $J$ Rehabil Med 2003; 41(Suppl): 60-65.

18 Johnson RL, Gerhart KA, McCray J, Menconi JC, Whiteneck GG. Secondary conditions following spinal cord injury in a population-based sample. Spinal Cord 1998; 36: 45-50.

19 Levi R, Hultling C, Seiger A. The Stockholm Spinal Cord Injury Study: 2. Associations between clinical patient characteristics and post-acute medical problems. Paraplegia 1995; 33: 585-594.

20 Krawetz P, Nance P. Gait analysis of spinal cord injured subjects: effects of injury level and spasticity. Arch Phys Med Rehabil 1996; 77: 635-638.

21 Norman KE, Pepin A, Barbeau H. Effects of drugs on walking after spinal cord injury. Spinal Cord 1998; 36: 699-715.

22 Sehgal N, McGuire JR. Beyond Ashworth. Electrophysiologic quantification of spasticity. Phys Med Rehabil Clin N Am 1998; 9: 949-979 ix..

23 Ashworth B. Preliminary trial of carisoprodol in multiple sclerosis. Practitioner 1964; 192: 540-542.

24 Haas BM, Bergstrom E, Jamous A, Bennie A. The inter rater reliability of the original and of the modified Ashworth scale for the assessment of spasticity in patients with spinal cord injury. Spinal Cord 1996; 34: 560-564.

25 McKay WB, Lim HK, Priebe MM, Stokic DS, Sherwood AM. Clinical neurophysiological assessment of residual motor control in post-spinal cord injury paralysis. Neurorehabil Neural Repair 2004; 18: 144-153.

26 Ben Smail D, Kiefer C, Bussel B. Clinical evaluation of spasticity. Neurochirurgie 2003; 49: 190-198.

27 Pierson SH. Outcome measures in spasticity management. Muscle Nerve Suppl 1997; 6: S36-S60.
28 Sherwood AM, Graves DE, Priebe MM. Altered motor control and spasticity after spinal cord injury: subjective and objective assessment. J Rehabil Res Dev 2000; 37: 41-52.

29 Priebe MM, Sherwood AM, Thornby JI, KharasNF, Markowski J. Clinical assessment of spasticity in spinal cord injury: a multidimensional problem. Arch Phys Med Rehabil 1996; 77: 713-716.

30 Sherrington CC. Flexion-reflex of the limb, crossed extension-reflex, and reflex stepping and standing. J Physiol (Lond) 1910; 40: 28-121.

31 Ditunno JF, Little JW, Tessler A, Burns AS. Spinal shock revisited: a four-phase model. Spinal Cord 2004; 42: 383-395.

32 Bennett DJ, Li Y, Siu M. Plateau potentials in sacrocaudal motoneurons of chronic spinal rats, recorded in vitro. J Neurophysiol 2001; 86: 1955-1971.

33 Noth J. Trends in the pathophysiology and pharmacotherapy of spasticity. J Neurol 1991; 238: 131-139.

34 Gracies JM, Nance P, Elovic E, McGuire J, Simpson DM. Traditional pharmacological treatments for spasticity. Part II: General and regional treatments. Muscle Nerve Suppl 1997; 6: S92-120.

35 Mayer NH. Clinicophysiologic concepts of spasticity and motor dysfunction in adults with an upper motoneuron lesion. Muscle Nerve Suppl 1997; 6: S1-13.

36 Dietz V. Spinal cord lesion: effects of and perspectives for treatment. Neural Plast 2001; 8: 83-90.

37 Beres-Jones JA, Johnson TD, Harkema SJ. Clonus after human spinal cord injury cannot be attributed solely to recurrent muscle-tendon stretch. Exp Brain Res 2003; 149: 222-236.

38 Rossi A, Mazzocchio R, Scarpini C. Clonus in man: a rhythmic oscillation maintained by a reflex mechanism. Electroencephalogr Clin Neurophysiol 1990; 75: 56-63.

39 Schmit BD, Benz EN, Rymer WZ. Reflex mechanisms for motor impairment in spinal cord injury. Adv Exp Med Biol 2002; 508: 315-323.

40 Schmit BD, McKenna-Cole A, Rymer WZ. Flexor reflexes in chronic spinal cord injury triggered by imposed ankle rotation. Muscle Nerve 2000; 23: 793-803.

41 Hornby TG, Rymer WZ, Benz EN, Schmit BD. Windup of flexion reflexes in chronic human spinal cord injury: a marker for neuronal plateau potentials? $J$ Neurophysiol 2003; 89: 416-426.

42 Gracies JM, Elovic E, McGuire J, Simpson DM. Traditional pharmacological treatments for spasticity. Part I: Local treatments. Muscle Nerve Suppl 1997; 6: S61-S91.

43 Albert T, Yelnik A. Physiotherapy for spasticity. Neurochirurgie 2003; 49: 239-246.

44 Barolat G, Singh-Sahni K, Staas Jr WE, Shatin D, Ketcik B, Allen K. Epidural spinal cord stimulation in the management of spasms in spinal cord injury: a prospective study. Stereotact Funct Neurosurg 1995; 64: 153-164.

45 Pinter MM, Gerstenbrand F, Dimitrijevic MR. Epidural electrical stimulation of posterior structures of the human lumbosacral cord: 3. Control Of spasticity. Spinal Cord 2000; 38: 524-531.

46 Midha M, Schmitt JK. Epidural spinal cord stimulation for the control of spasticity in spinal cord injury patients lacks long-term efficacy and is not cost-effective. Spinal Cord 1998; 36: 190-192.

47 Mirbagheri MM, Ladouceur M, Barbeau H, Kearney RE. The effects of long-term FES-assisted walking on intrinsic 
and reflex dynamic stiffness in spastic spinal-cord-injured subjects. IEEE Trans Neural Syst Rehabil Eng 2002; 10: 280-289.

48 Rode G, Maupas E, Luaute J, Courtois-Jacquin S, Boisson D. Medical treatment of spasticity. Neurochirurgie 2003; 49: 247-255.

49 Rang HP, DaleMM, Ritter JM, Gardner P. Pharmacology. Churchill Livingstone: New York 1995.

50 Stewart JE, Barbeau H, Gauthier S. Modulation of locomotor patterns and spasticity with clonidine in spinal cord injured patients. Can J Neurol Sci 1991; 18: 321-332.

51 Fung J, Stewart JE, Barbeau H. The combined effects of clonidine and cyproheptadine with interactive training on the modulation of locomotion in spinal cord injured subjects. J Neurol Sci 1990; 100: 85-93.

52 Wainberg $M$, Barbeau $\mathrm{H}$, Gauthier S. The effects of cyproheptadine on locomotion and on spasticity in patients with spinal cord injuries. J Neurol Neurosurg Psychiatr 1990; 53: 754-763.

53 Korenkov AI, Niendorf WR, Darwish N, Glaeser E, Gaab MR. Continuous intrathecal infusion of baclofen in patients with spasticity caused by spinal cord injuries. Neurosurg Rev 2002; 25: 228-230.

54 Emery E. Intrathecal baclofen. Literature review of the results and complications. Neurochirurgie 2003; 49: 276-288.

55 Barnes M. Botulinum toxin - mechanisms of action and clinical use in spasticity. $J$ Rehabil Med 2003; 41(Suppl): $56-59$.

56 Al Khodairy AT, Gobelet C, RossierAB. Has botulinum toxin type A a place in the treatment of spasticity in spinal cord injury patients? Spinal Cord 1998; 36: 854-858.

57 Chambers HG. The surgical treatment of spasticity. Muscle Nerve Suppl 1997; 6: S121-S128. 